


\section{DISCLAIMER}

This report was prepared as an account of work sponsored by an agency of the United States Government. Neither the United States Government nor any agency thereof, nor any of their employees, makes any warranty, express or implied, or assumes any legal liability or responsibility for the accuracy, completeness, or usefulness of any information, apparatus, product, or process disclosed, or represents that its use would not infringe privately owned rights. Reference herein to any specific commercial product, process, or service by trade name, trademark, manufacturet, or otherwise does not necessarily constitute or imply its endorsement, recommendation, or favoring by the United States Government or any agency thereof. The views and opinions of authors expressed herein do not necessarily state or reflect those of the United States Government or any agency thereof. 


\title{
On Limited Fan-in Optimal Neural Networks
}

\author{
Valeriu Beiu $^{1,2}$, Sorin Draghici ${ }^{3}$ and Hanna E. Makaruk ${ }^{4,5}$ \\ ${ }^{1}$ Space and Atmospheric Division NIS-1, MS D466, Los Alamos National Laboratory \\ Los Alamos, New Mexico 87545, USA \\ E-mail: beiualanl.gov \\ ${ }^{3}$ Vision and Neural Networks Laboratory, Department of Computer Science \\ Wayne State University, 431 State Hall, Detroit, MI 48202, USA \\ E-mail: sod@cs . wayne. edu \\ 4 Theoretical Division T-13, MS B213, Los Alamos National Laboratory \\ Los Alamos, New Mexico 87545, USA \\ E-mail: hanna@t13.lanl.gov
}

\begin{abstract}
Because VLSI implementations do not cope well with highly interconnected nets-the area of a chip growing as the cube of the fan-in [25]-this paper analyses the influence of limited fan-in on the size and VLSI optimality of such nets.

Two different approaches will show that VLSI- and sizeoptimal discrete neural networks can be obtained for small (i.e. lower than linear) fan-in values. They have applications to hardware implementations of neural networks. The first approach is based on implementing a certain sub-class of Boolean functions, $\boldsymbol{F}_{n, m}$ functions [34]. We will show that this class of functions can be implemented in.VLSI-optimal (i.e., minimising $A T^{2}$ ) neural networks of small constant fan-ins. The second approach is based on implementing Boolean functions for which the classical Shannon's decomposition can be used. Such a solution has already been used to prove bounds on neural networks with fan-ins limited to 2 [26]. We will generalise the result presented there to arbitrary fan-in, and prove that the size is minimised by small fan-in values, while relative minimum size solutions can be obtained for fan-ins strictly lower than linear.

Finally, a size-optimal neural network having small constant fan-ins will be suggested for $\mathbb{F}_{n, m}$ functions.

Keywords-neural networks, VLSI, fan-in, Boolean circuits, threshold circuits, $\mathbb{F}_{n, m}$ functions.

2. 'On leave of absence' from the "Politehnica" University of Bucharest, Computer Science Department, Spl. Independentei 313, RO-77206 Bucharest, România.

5

On leave of absence from the Polish Academy of Sciences, Institute of Fundamental Technological Research, Swietokrzyska 21, 00-049 Warsaw, Poland.
\end{abstract}

\section{Introduction}

In this paper we shall consider feedforward neural networks (NNs) made of linear threshold gates (TGs). A neuron (i.e., linear TG) will compute a Boolean function (BF) $f:\{0,1\}^{n} \rightarrow\{0,1\}$, where one of the $k$ input vectors is $Z_{k}=$ $\left(z_{k, 0}, \ldots, z_{k, n-1}\right) \in\{0,1\}^{n}$ and $f\left(Z_{k}\right)=\operatorname{sgn}\left(\sum_{i=0}^{n-1} w_{i} z_{k, i}+\theta\right)$, with the synaptic weights $w_{i} \in \mathbb{R}$, thresholds $\theta \in \mathbb{R}$, and $s g n$ the sign function. Two cost functions commonly associated to a $\mathrm{NN}$ are: $(i)$ depth, which is the number of layers (or the number of edges-if we consider unit length for all the edges connecting the TGs) on the longest input to output path; and (ii) size (or node complexity), which is the number of neurons (TGs). Unfortunately, these measures are not the best criteria for ranking different solutions when going for silicon [21], as "comparing the number of nodes is inadequate for comparing the complexity of neural networks as the nodes themselves could implement quite complex functions" [41].

The fact that size is not a good estimate for area is explained as: ( $i$ ) the area (of one neuron) can be related to its associated weights, and (ii) the area of the connections isin most cases-neglected. Here are several alternatives of how the area could scale in relation to weights and thresholds:

- for purely digital implementation, the area scales at least with the cumulative size of the weights and thresholds (the bits for representing these weights and thresholds have to be stored);

- for certain types of analog implementations (e.g., using resistors or capacitors), the same type of scaling 
is valid (in particular cases, analog implementations can have binary encoding, thus the area would scale with the cumulative log-scale size of the parameters);

- there are some types of implementations (e.g., transconductance ones) which offer a constant size per element, thus scaling only with $\Sigma_{N N}$ fan-ins.

All these 'cost functions' are linked to VLSI by the assumptions one makes on how the area of a chip scales with the weights and the thresholds $[5,9,10]$. That is why several other measures (i.e., 'cost functions')—beside sizehave already been used:

- the total number-of-connections, or $\Sigma_{N N}$ fan-ins, has been used by several authors $[1,25,30,33]$;

- the total number-of-bits needed to represent the weights and thresholds $\Sigma_{N N}\left(\sum_{i}\left\lceil\log \mid w_{i} \|+\lceil\log \mid \theta 1\rceil\right)^{1}\right.$ has been used by others $[22,23,41]$;

- the sum of all the absolute values of the weights and thresholds $\Sigma_{N N}\left(\Sigma_{i}\left|w_{i}\right|+|\theta|\right)$ has also been advocated $[5,10,18,19,21]$, while another similar 'cost function' is $\Sigma_{N N}\left(\Sigma_{i} w_{i}{ }^{2}+\theta^{2}\right)$, which has been used in the context of genetic programming for reaching minimal NNs [44].

The sum of all the absolute values of the weights and thresholds has also been used as an optimum criterion for: (i) linear programming synthesis [32]; (ii) defining the minimum-integer TG realisation of a function [27]. Recently [3], the same measure (under the name of "total weight magnitude") has been used in the context of computational learning theory. It was proven that the generalisation error of NNs used for classification depends on the size of the weights-rather than the number of weights-by showing that the misclassification probability converges at a rate of $O\{(c A) l \sqrt{m}\}$. Here $A$ is the sum of the magnitude of the weights, $l$ is the depth, $m$ is the number of examples, and $c$ is a constant.

With respect to delay, two VLSI models have been commonly in use [38]:

- the simplest one assumes that delay is proportional to the input capacitance, hence a TG introduces a delay proportional to its fan-in;

- a more exact one considers the capacitance along any wire, hence the delay is proportional to the length of the connecting wires.

It is worth emphasising that it is anyhow desirable to limit the range of parameter values [42] for VLSI implementations--be they digital or analog-because both the maximum value of the fan-in $[28,40]$ and the maximal ratio between the largest and the smallest weight $[23,24,29,42]$ cannot grow over a certain (technological) limit.

1 In this paper $\lfloor x\rfloor$ is the floor of $x$, i.e. the largest integer less than or equal to $x$, and $\lceil x\rceil$ is the ceiling of $x$, i.e. the smallest integer greater or equal to $x$. In this paper all the logarithms are to the base 2, except explicitly mentioned otherwise.
The focus of this paper will be on NNs having limited fan-in (the fan-in will be denoted by $\Delta$ ). We will present both theoretical proofs and simulations in support of our claim that VLSI- and size-optimal NNs can be obtained for small fan-ins. For simplification, we shall consider only NNs having $n$ binary inputs and $\mu$ binary outputs (if real inputs and outputs are needed, it is always possible to quantize them up to a certain number of bits such as to achieve a desired precision $[4,7,11,24])$. In Section 2 we shall present a theoretical proof for the $\mathbb{F}_{n, m}$ class of functions, showing that their VLSI-optimal implementation is achieved with small constant fan-ins. Section 3 deals with $\mathrm{BFs}$, and details the generalisation of a result from [26] to arbitrary fan-ins. Based on that generalisation we will show that the size can be minimised for small fan-ins. Finally, in Section 4 we will suggest how to implement $\boldsymbol{F}_{n, m}$ functions in size-optimal NNs having small constant fan-ins.

Conclusions, open questions and further directions for research complete the paper. Due to space limitations some of the lengthy mathematical proofs suggested in $[6,7]$ have been omitted, but can be found in $[9,10]$.

\section{VLSI-optimal neural implementations of IF $F_{n, m}$ functions}

$\mathbb{F}_{n, m}$ is the class of BFs of $n$ variables having $m$ groups of ones in their truth table. Obviously, any BF can be represented by a suitable collection of its true values (ones), but for achieving that the number of groups of ones grows exponentially (i.e. $\boldsymbol{F}_{n, 2^{n / 2}}$ completely covers $\boldsymbol{B}_{n}$, the set of all $n$-ary BFs). This class of functions has been introduced and analysed by Red'kin [34]; he constructively proved that a depth-3 NN has size .

Proposition 1 (Theorem 1 from [34]) The complexity realisation (i.e., number of threshold elements) of $I F_{n, m}$ (the class of Boolean functions $f\left(x_{1} x_{2} \ldots x_{n-1} x_{n}\right)$ that have exactly $m$ groups of ones) is at most $2 \sqrt{2 m}+3$.

The construction has: a first layer of $\left\lceil(2 m)^{1 / 2}\right\rceil$ TGs (COMPARISONs) with fan-in $=n$ and weights $\leq 2^{n-1}$; a second layer of $2\left\lceil(m / 2)^{1 / 2}\right\rceil$ TGs of fan-in $=n+\left\lceil(2 m)^{1 / 2}\right\rceil$ and weights $\leq 2^{n}$; one more TG of fan-in $=2\left\lceil(\mathrm{~m} / 2)^{1 / 2}\right\rceil$ and weights $\in\{-1,+1\}$ in the third layer.

Red'kin also proved that if the implementation of BFs of this type is restricted to circuits having no more than three layers, than the upper bound-following his method of synthesis-is equal to the lower bound obtained from capacity considerations. Although this construction is sizeoptimal, it is not VLSI-optimal as the weights and thresholds are exponential.

Another solution was detailed in $[20,21]$ and later improved in $[5,15,16]$. It has a first layer of COMPARISONs 
- followed by a second layer of MAJORITY gates. This solution relies on the classical COMPARISON of two binary numbers $\boldsymbol{X}=x_{n-1} x_{n-2} \ldots x_{1} x_{0}$ and $\boldsymbol{Y}=y_{n-1} y_{n-2} \ldots y_{1} y_{0}$, which is a $B F$ defined as:

$$
C_{n}^{>(\geq)}=C_{n}^{>(\geq)}(X, Y)=\left\{\begin{array}{llll}
1 & \text { if } & X>Y & (X \geq Y) \\
0 & \text { if } & X \leq Y & (X<Y)
\end{array} .\right.
$$

It is known from previous work that COMPARISON cannot be computed by a single TG with polynomially bounded integer weights, but can be computed by a depth- $2 \mathrm{NN}$ with $O\left(n^{4}\right)$ TGs and polynomially bounded weights [2]. This last result has been improved as follows.

Proposition 2 (Lemma 6 [37]) The COMPARISON function can be computed in a depth-3 neural network of size $3 n$ with polynomially bounded integer weights.

This constructive solution (we shall call it SRK) has a first layer of $n$ AND gates computing $x_{i} \wedge \bar{y}_{i}$, and $n$ OR gates computing $x_{i} \vee \bar{y}_{i}$, followed by a layer of $n-1$ AND gates:

$$
B_{k}=\left(x_{k} \wedge \bar{y}_{k}\right) \wedge\left\{\wedge_{j=k+1}^{n-1}\left(x_{j} \vee \bar{y}_{j}\right)\right\},
$$

and a third layer having one $O R$ gate:

$$
C_{n}^{\geq}=\left(x_{n-1} \wedge \bar{y}_{n-1}\right) \vee\left(\vee_{k=0}^{n-2} B_{k}\right) .
$$

This depth-3 NN has size SaK $=3 n-1$, thresholds $\leq n$, fanin $\leq n$, and all the weights \pm 1 .

Proposition 3 (Proposition 2 and 3 [7]) The COMPARISON of two n-bit numbers can be computed by $a \Delta$-ary tree neural network having integer weights and thresholds which are: (i) polynomially bounded for all the values of the fan-in $3 \leq \Delta \leq \boldsymbol{O}(\log n)$; (ii) super-polynomially bounded for all the values of the fan-in $O(\log n)<\Delta \leq \boldsymbol{O}\left(\log ^{k} n\right)$; and (iii) exponentially bounded for all the values of the fan-in $o\left(\log { }^{k} n\right)<\Delta \leq 2 n$.

This constructive class of solutions (which we shall call
B_A), was proposed in $[18,19]$, and is based on decomposing COMPARISON in a tree structure. The NN has a first layer of 'partial' COMPARISONs $\boldsymbol{C}_{\Delta}^{>}$and $\boldsymbol{C}_{\Delta}^{2}([\Delta / 2\rfloor$ bits from $\boldsymbol{X}$ and $[\Delta / 2\rfloor$ bits from $Y)$ followed by a $\Delta$-ary tree of TGs combining these partial results. The fact that the BFs implemented by the nodes are linear separable functions was proven in $[17,21]$. The network has:

$$
\begin{aligned}
& \operatorname{depth}_{\mathbf{B}_{-} \Delta}=\lceil\log n /(\log \Delta-1)\rceil \text {, and } \\
& \text { size }_{\mathrm{B}_{-} \Delta}=\lceil 4(n-1) / \Delta-2\rceil-\text { depth }_{\mathrm{B} \_\Delta}
\end{aligned}
$$

with fan-in of $\Delta$ or $\Delta-1$. The weights and the thresholds are lower than $2^{\Delta / 2}$ (for $3 \leq \Delta \leq 2 n$ ).

Proposition 4(Theorem 3 [35]) The size complexity of COMPARISON implemented by generalised symmetric functions is $\Theta(n / \log n)$.

This constructive solution (we shall call it Ros) has a first layer of 'partial' COMPARISONs $C_{i}$ (equivalent to $\boldsymbol{C}_{2 m}^{>}$) and $\tilde{C}_{i}$ (equivalent to $C_{2 m}^{\geq}$) having $m$ input bits from $\boldsymbol{X}$ and $m$ input bits from $Y$. The first layer has $2\lceil n / m\rceil-1$ TGs of fan-in $=2 m$. The second layer has $[n / m]-1$. AND gates with fan-in $=2,3, \ldots,\lceil n / m\rceil$ :

$$
B_{k}=\tilde{C}_{k} \wedge\left(\wedge_{j=k+1}^{\lceil n / m\rceil} C_{j}\right) .
$$

The third layer has one OR gate $C_{n}^{\geq}(X, Y)=\vee_{k=1}^{[n / m]} B_{k}$, and by taking $m=\lceil\log n\rceil+1$, size $e_{\text {Ros }}=3\lceil n /(\lceil\log n\rceil+1)\rceil-1$. The NN has depth-3, weights and thresholds $\leq 2^{\lceil\log n\rceil}$ and fan-in $\leq\lceil n /(\lceil\log n\rceil+1)\rceil$.

Proposition 5 (Corollary 2 [39]) The COMPARISON can be computed by a depth-2 linear threshold network of size $2\left\lceil n /\lceil\sqrt{n}\rceil\right.$, with weight values at most $2^{\lceil\sqrt{n}\rceil}$ and with an upper bound of $2\lceil\sqrt{n} 1+1$ for the maximum fan-in.

This constructive solution (we shall call it vCB) has a

\begin{tabular}{|c|c|c|c|c|c|c|c|c|}
\hline \multirow{2}{*}{\multicolumn{2}{|c|}{ Length }} & vсB [39] & B_2 $\lceil\sqrt{n}\rceil[6]$ & SRK [37] & ROs [35] & vсв [39] & B_s $[18,19]$ & \multirow{2}{*}{$\frac{B \_\Delta[18,19]}{\text { depth }=4}$} \\
\hline & & \multicolumn{2}{|c|}{ depth $=2$} & \multicolumn{4}{|c|}{ depth $=3$} & \\
\hline \multirow[t]{3}{*}{ 32-bits } & Size & 13 & 12 & 95 & 19 & 18 & 19 & 45 \\
\hline & Max. weight & 64 & 32 & 32 & 64 & 16 & 8 & 3 \\
\hline & Max. fan-in & 13 & 12 & 32 & 12 & 9 & 8 & 5 \\
\hline \multirow[t]{3}{*}{ 64-bits } & Size & 17 & 16 & 191 & 31 & 26 & 39 & 63 \\
\hline & Max. weight & 256 & 128 & 64 & 128 & 64 & 8 & 5 \\
\hline & Max. fan-in & 17 & 16 & 64 & 14 & 13 & 8 & 7 \\
\hline
\end{tabular}
first layer of 'partial' COMPARISONs $(\lceil\sqrt{n}\rceil$ input bits from $X$ and $(\sqrt{n}\rceil$ input bits from $Y$ ), and the second layer computes the carry-out of the 2-1 binary ADDITION with carry.

Table 1 (from [7])

Size, weights and fan-ins of different solutions for COMPARISON (normal operand lengths). 
Table 2

Different estimates for the $A T^{2}$ of COMPARISON for SRK, B_4, B_log, ROS and VCB.

\begin{tabular}{|c|c|c|c|}
\hline Area Delay & Depth & Fan-in & Length \\
\hline \multirow[t]{2}{*}{ Size } & $A T_{\mathrm{VCB}}^{2}=O(\sqrt{n})$ & $A T_{B_{-} 4}^{2}=O\left(n \log ^{2} n\right)$ & $A T_{\mathrm{VCB}}^{2}=O\left(n^{2} \sqrt{n}\right)$ \\
\hline & $\begin{array}{l}A T_{\mathrm{ROS}}^{2}=\boldsymbol{O}(n / \log n) \\
A T_{\mathrm{SAK}}^{2}=O(n) \\
A T_{\mathrm{B}-\mathrm{LOg}}^{2}=O\left[n \log n / \log ^{2}(\log n)\right] \\
A T_{\mathrm{B} 4}^{2}=O\left(n \log ^{2} n\right)\end{array}$ & $\begin{array}{l}A T_{\mathrm{B}-\log }^{2}=O\left[n \log ^{3} n / \log ^{2}(\log n)\right] \\
A T_{\mathrm{VCB}}^{2}=O(n \sqrt{n}) \\
A T_{\mathrm{ROS}}^{2}=O\left(n^{3} / \log ^{3} n\right) \\
A T_{\mathrm{SRK}}^{2}=O\left(n^{3}\right)\end{array}$ & $\begin{array}{l}A T_{\text {ROS }}^{2} \cong 3 \cdot n^{3} / \log n \\
A T_{\mathrm{B}_{-} \log }^{2} \cong 4 \cdot n^{3} / \log n \\
A T_{\mathrm{B}_{-} 4} \cong 4 \cdot n^{3} \\
A T_{\mathrm{SRK}}^{2} \cong 27 n^{3} / 4 \\
\end{array}$ \\
\hline \multirow[t]{3}{*}{$\Sigma_{N N}$ fan-ins } & $A T_{\mathrm{VCB}}^{2}=O(n)$ & $A T_{\mathrm{B}_{-4}}^{2}=O\left(n \log ^{2} n\right)$ & \multirow{2}{*}{$\begin{array}{l}A T_{\mathrm{B} \_ \text {log }}^{2} \cong 4 n^{3} \\
A T_{\mathrm{VCB}}^{2} \cong 4 n^{3} \\
A T_{\mathrm{B} 4}^{2} \cong 5 n^{3}\end{array}$} \\
\hline & \multirow{2}{*}{$\begin{array}{l}A T_{\mathrm{B}-\log }^{2}=\boldsymbol{O}\left[n \log ^{2} n / \log ^{2}(\log n)\right] \\
A T_{\mathrm{B}-4}^{2}=\boldsymbol{O}\left(n \log ^{2} n\right) \\
A T_{\mathrm{ROS}}^{2}=O\left(n^{2} / \log ^{2} n\right) \\
A T_{\text {SRK }}^{2}=O\left(n^{2}\right)\end{array}$} & \multirow{2}{*}{$\begin{array}{l}A T_{\mathrm{B}_{-} \log }^{2}=\boldsymbol{O}\left[n \log ^{4} n / \log ^{2}(\log n)\right] \\
A T_{\mathrm{VCB}}^{2}=\boldsymbol{O}\left(n^{2}\right) \\
A T_{\mathrm{ROS}}^{2}=\boldsymbol{O}\left(n^{4} / \log ^{4} n\right) \\
A T_{\mathrm{SRK}}^{2}=\boldsymbol{O}\left(n^{4}\right)\end{array}$} & \\
\hline & & & $\begin{array}{l}A T_{\text {ROS }}^{2}=\boldsymbol{O}\left(n^{4} / \log ^{2} n\right) \\
A T_{\text {SRK }}^{2}=\boldsymbol{O}\left(n^{4}\right)\end{array}$ \\
\hline \multirow[t]{2}{*}{$\sum_{N N}\left(\sum_{i}\left|w_{i}\right|+|\theta|\right)$} & $A T_{\mathrm{B}_{-4}}^{2}=O\left(n \log ^{2} n\right)$ & $A T_{B_{-} 4}^{2}=O\left(n \log ^{2} n\right)$ & $A T_{\mathrm{B}-4}^{2}=O\left(n^{3}\right)$ \\
\hline & $\begin{array}{l}A T_{\mathrm{B}_{2} \log }^{2}=\boldsymbol{O}\left[n \sqrt{n} \log n / \log ^{2}(\log n)\right] \\
A T_{\mathrm{FOS}}^{2}=\boldsymbol{O}\left(n^{2} / \log n\right) \\
A T_{\mathrm{SRK}}^{2}=\boldsymbol{O}\left(n^{2}\right) \\
A T_{\mathrm{VCB}}^{2}=\boldsymbol{O}\left(n^{1 / 2} 2^{\sqrt{n}}\right)\end{array}$ & $\begin{array}{l}A T_{\mathrm{B}-\log }^{2}=O\left[n \sqrt{n} \log ^{3} n / \log ^{2}(\log n)\right] \\
A T_{\mathrm{ROS}}^{2}=O\left(n^{4} / \log ^{3} n\right) \\
A T_{\mathrm{SRK}}^{2}=O\left(n^{4}\right) \\
A T_{\mathrm{VCB}}^{2}=O\left(n^{3 / 2} 2^{\sqrt{n}}\right)\end{array}$ & $\begin{array}{l}A T_{\mathrm{B} \log }^{2}=\boldsymbol{O}\left(n^{3} \sqrt{n} / \log n\right) \\
A T_{\mathrm{ROS}}^{2}=\boldsymbol{O}\left(n^{4} / \log n\right) \\
A T_{\mathrm{SRK}}^{2}=O\left(n^{4}\right) \\
A T_{\mathrm{VCB}}^{2}=O\left(n^{5 / 2} 2^{\sqrt{n}}\right)\end{array}$ \\
\hline
\end{tabular}

Although size-optimal (like [34]), this solution is not very attractive from the VLSI complexity point of view as having exponentially growing weights. The complexity results are similar to those of a particular solution belonging to the B_ $\Delta$ class $[6,7]$ : take $\Delta=2\lceil\sqrt{n}\rceil$ 一 which leads to $B \_2[\sqrt{n}\rceil-$ and the NN has depth $=2$, size $=\lceil 2 \sqrt{n}\rceil$, with weights and thresholds of at most $2^{\sqrt{n} 7}$.

For normal length COMPARISONs Vassiliadis et al. [39] claim improvements over ROS [35] and SRK [37]. We present in Table 1 the results reported in [7]. Both VCB and B_2 $[\sqrt{n}\rceil$ achieve better performances than SRK and ROS. For depth $=2$, B_2 $[\sqrt{n} 7$ outperforms VCB (both for 32-bit and for 64-bit operand lengths), while for depth $=3, B \_2[\sqrt{n}\rceil$ has lower weights and fan-ins, but slightly more gates. Still, B_ $\Delta$ has two main advantages: (i) being a class of solutions it can be used for other depths (see the last column of Table I); (ii) because the weights and the fan-ins are lower, the area should also be lower.

It is known that a VLSI design is considered optimum when judged on a combined measure $A T^{2}$ [38], thus:

$$
A T_{\mathrm{SRK}}^{2}=(3 n-1) \times 3^{2}=O(n)
$$

$$
\begin{aligned}
& A T_{\mathrm{B}_{-} \Delta}^{2}<\left\lceil\frac{4(n-1)}{\Delta-2}\right\rceil \times\left[\frac{\log n}{\log \Delta-1}\right]^{2}=O\left(n \log ^{2} n / \Delta \log \Delta\right) \\
& A T_{\mathrm{ROS}}^{2}=3\lceil n /(\lceil\log n\rceil+1)\rceil \times 3^{2}=O(n / \log n) .
\end{aligned}
$$

The natural extension of the circuit complexity results to VLSI complexity ones is by using the closer estimates for the area and the delay (as discussed in Introduction).

Proposition 6 If the area of a neural network is estimated as $\Sigma_{N N}$ fan-ins, there are neural networks computing the COMPARISON of two n-bit numbers which occupy between $\boldsymbol{O}(n)$ and $\boldsymbol{O}\left(n^{2}\right)$ area:

$$
\begin{aligned}
A_{\mathrm{SRK}}=\left(n^{2}+11 n-6\right) / 2 & =O\left(n^{2}\right) \\
A_{\mathrm{B}_{\triangle} \Delta}<\Delta \times\left\lceil\frac{4(n-1)}{\Delta-2}\right\rceil+\left\lceil\frac{\log n}{\log \Delta-1}\right\rceil+\left\lceil\frac{4 n}{\Delta}\right\rceil=O(n) & \left.O=\frac{n}{2}\right]^{2}+\frac{3}{2} \cdot\left[\frac{n}{\lceil\log n\rceil+1}\right\rceil \\
\left.A_{\mathrm{ROS}}<4 n+\frac{1}{\lceil\log n\rceil+1}\right]^{2} & =O\left(n^{2} / \log ^{2} n\right) .
\end{aligned}
$$



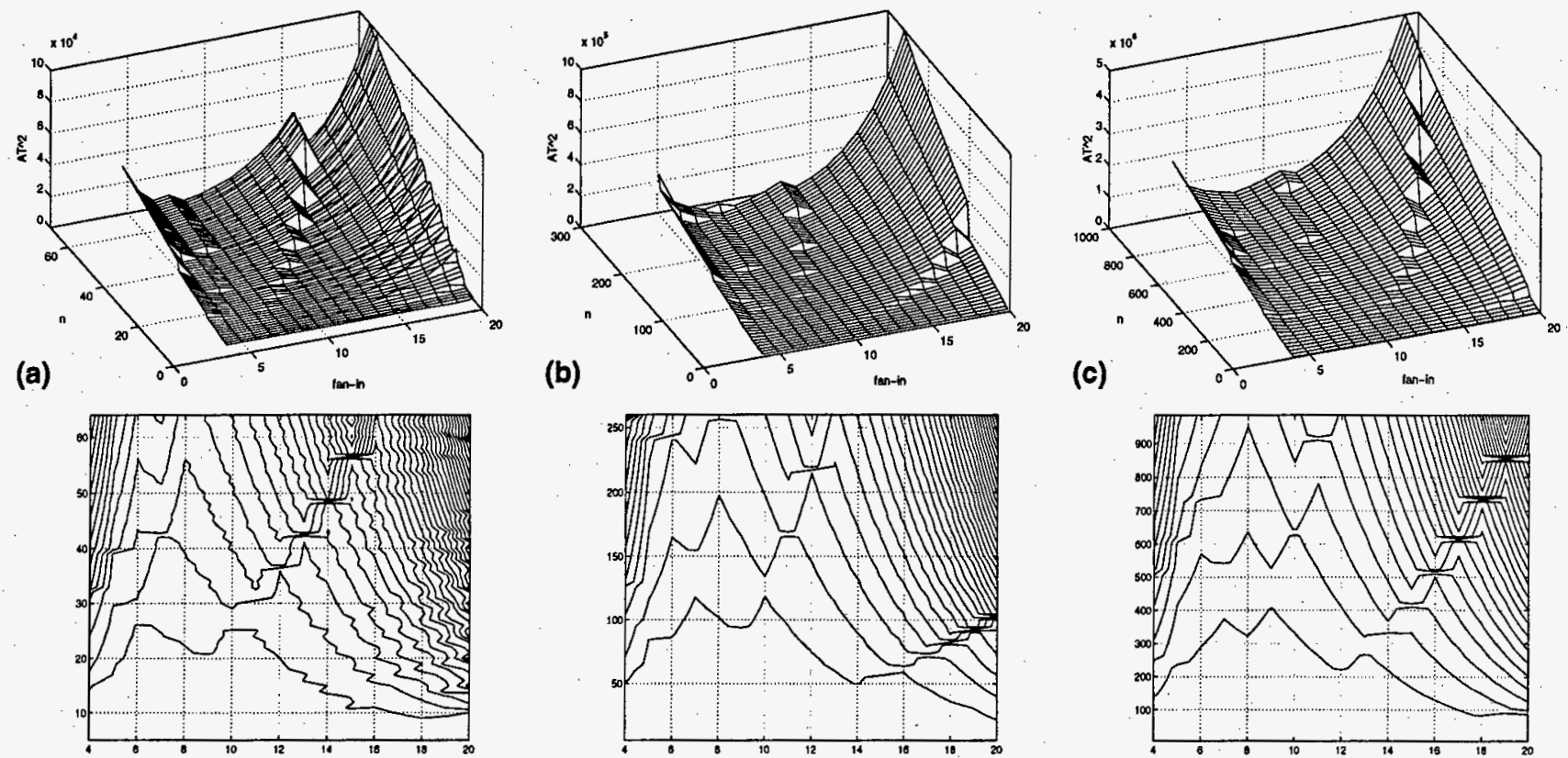

(d)

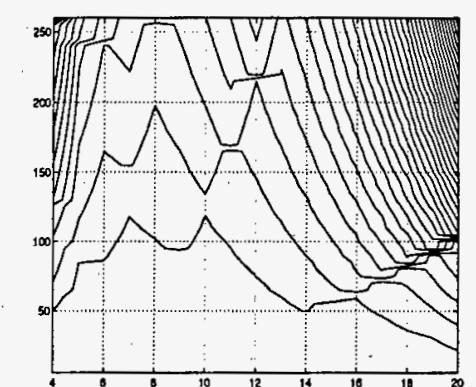

(e)

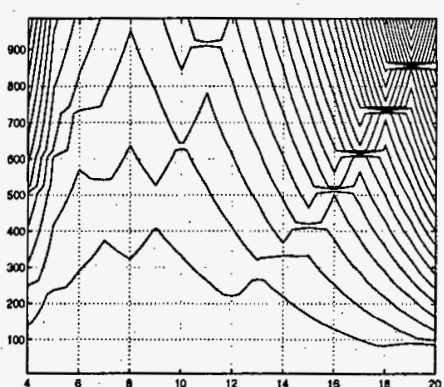

(f)

Figure 1. The $A T^{2}$ values of COMPARISON-plotted as a 3D surface-versus the number of inputs $n$ and the fan-in $\Delta$ for $4 \leq \Delta \leq 20$ : (a) $n \leq 64$; (b) $n \leq 256$; (c) $n \leq 1024$; (d), (e), and (f) show the contour plots for the same cases. Clearly a 'valley' is formed, and that the 'deepest' points constantly lie somewhere between $\Delta$ minim $=6$ and $\Delta$ maxim $=9$.

Proposition 7 If the area of a neural network is estimated as $\Sigma_{N N}\left(\Sigma_{i}\left|w_{i}\right|+|\theta|\right)$, there are neural networks computing the COMPARISON of two n-bit numbers which occupy between $O(n)$ and $O\left(n^{2}\right)$ area:

$$
\begin{aligned}
A_{\mathrm{SRK}}=\left(2 n^{2}+15 n-9\right) / 2 & =O\left(n^{2}\right) \\
A_{\mathrm{B} \_\Delta}<\frac{2^{\Delta / 2}}{\Delta} \cdot \frac{8 n \Delta-6 n-5 \Delta}{\Delta-2} & =O\left(n \cdot 2^{\Delta / 2} / \Delta\right) \\
A_{\mathrm{ROS}}<\left(4 n-\frac{5}{2}\right) \times\left\lceil\frac{n}{\lceil\log n\rceil+1}\right\rceil+ & {\left[\frac{n}{\lceil\log \eta\rceil+1}\right]^{2} } \\
& =O\left(n^{2} / \log n\right) .
\end{aligned}
$$

Proposition 8 If the delay of one neuron is proportional to its fan-in, there are neural networks computing the COMPARISON of two n-bit numbers which require between $\boldsymbol{O}(\log n)$ and $\boldsymbol{O}(n)$ time:

$$
\begin{aligned}
& T_{\text {SAK }}=2 n+2 \quad=O(n) \\
& T_{\mathrm{B}_{\_} \Delta}<(\Delta-1) \times\left[\frac{\log n}{\log \Delta-1}\right]+1=O(\Delta \log n / \log \Delta) \\
& T_{\mathrm{ROS}}<2 \times\left(\left\lceil\frac{n}{\lceil\log n\rceil+1}\right\rceil+\lceil\log n\rceil+1\right)=O(n / \log n) .
\end{aligned}
$$

Proposition 9 If the delay in a neural network is proportional to the length of the wires, there are neural networks computing the COMPARISON of two n-bit numbers which require $\boldsymbol{O}(n)$ time:

$$
T_{\mathrm{SRK}}=(3 n-1) / 2, \quad T_{\mathrm{B}_{-} \Delta}<n, \quad T_{\mathrm{ROS}}<n .
$$

For all these different estimations of $A$ and $T$, the $A T^{-2}$ complexity values have been computed, ordered, and can be seen in Table 2.

Not wanting to complicate the proof, we shall determine the VLSI-optimal fan-in when implementing COMPARISON (in fact an $\mathbb{F}_{n, 1}$ function) for which several solutions were detailed in Propositions 2 to 9. The same result is valid for $I_{n, m}$ functions as can be intuitively expected as:

- the delay is determined by the first layer of COMPARIsons; while

- the area is mostly influenced by the same first layer of COMPARISONs (the area for the implementing the MAJORITY gate can be neglected $[15,21])$.

From the alternatives presented in Table 2, we have chosen $\Sigma_{N N}\left(\Sigma_{i}\left|w_{i}\right|+|\theta|\right)$ for area and depth for delay, but other estimates lead to similar results (the optimal $A T^{2}$ being $O\left(n \log ^{2} n\right)$ in four out of nine cases-see Table 2). To get a better understanding, the $A T^{2}$ values have been computed for variable fan-ins and for different number of inputs $n$, and can be seen in Figure 1 . 


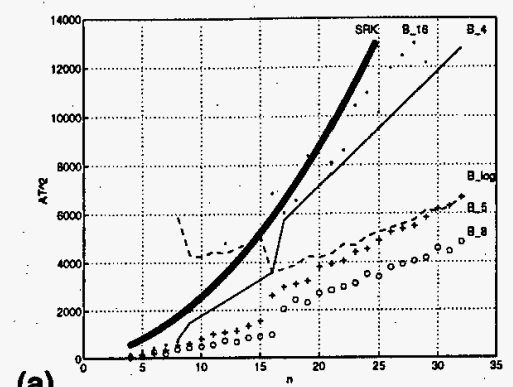

(a)

(b)

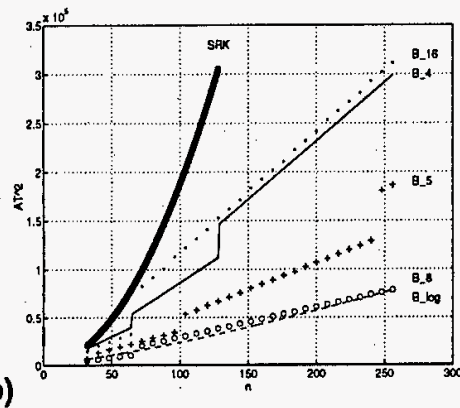

(e)
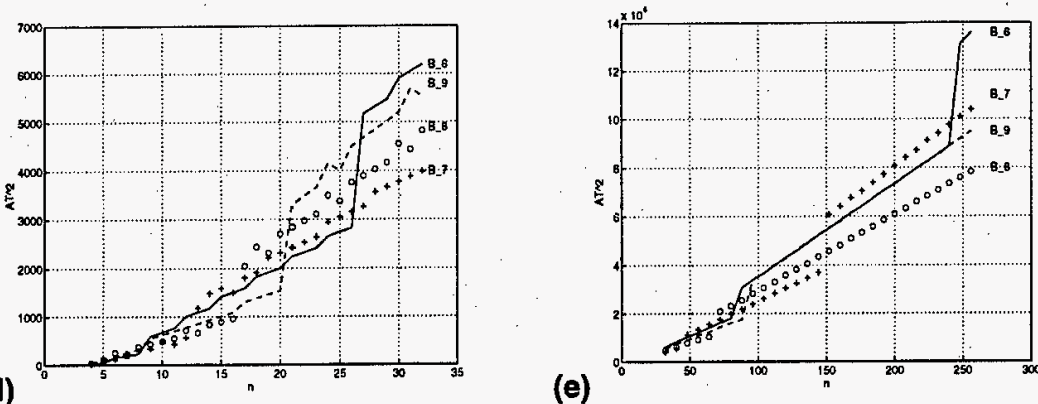

(c)

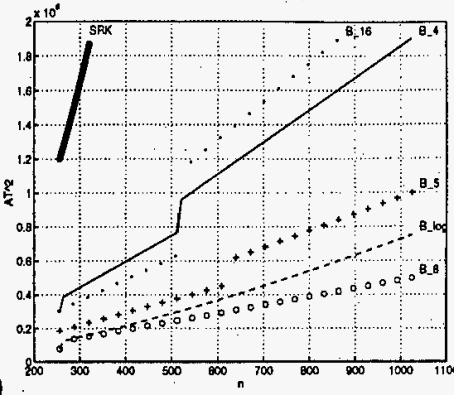

(f)

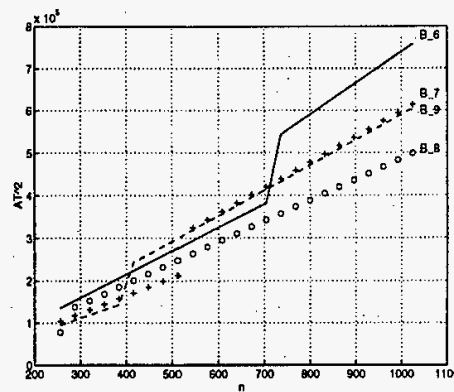

Figure 2. The $A T^{2}$ values of comPARISON for different number of inputs $\boldsymbol{n}$ and fan-in $\Delta$ for the intervals: (a) $4 \leq n \leq 32$; (b) $32 \leq n \leq 256$; and (c) $256 \leq n \leq 1024$. Details showing the 'optimum' fan-in for the same intervals: (d) $4 \leq n \leq 32$; (e) $32 \leq n \leq 256$; and (f) $256 \leq n \leq 1024$.

Proposition 10 The VLSI-optimal neural network which computes the COMPARISON of two n-bit numbers has smallconstant fan-in 'neurons' with small-constant bounded weights and thresholds.

Proof From Propositions 3 and 7:

$$
\begin{aligned}
A T^{2} & \equiv \frac{2^{\Delta / 2}}{\Delta}: \frac{8 n \Delta-6 n-5 \Delta}{\Delta-2} \cdot\left(\frac{\log n}{\log \Delta}\right)^{2} \\
& =O\left\{n \log ^{2} n \cdot 2^{\Delta / 2} /\left(\Delta \log ^{2} \Delta\right)\right\}
\end{aligned}
$$

and we compute the derivative:

$$
\begin{aligned}
\frac{d\left(A T^{2}\right)}{d \Delta}= & \frac{2^{\Delta / 2} \log ^{2} n}{\Delta^{2}(\Delta-2)^{2} \log ^{3} \Delta} \times\left(8 n \Delta^{3} \log \Delta-22 n \Delta^{2} \log \Delta\right. \\
& +12 n \Delta \log \Delta-5 \Delta^{3} \log \Delta+10 \Delta^{2} \log \Delta \\
& -\frac{16}{\ln 2} n \Delta^{2} \log \Delta+\frac{24}{\ln 2} n \Delta \log \Delta-\frac{24}{\ln 2} n \log \Delta \\
& +\frac{10}{\ln 2} \Delta^{2} \log \Delta-\frac{32}{\ln 2} n \Delta^{2}+\frac{88}{\ln 2} n \Delta-\frac{48}{\ln 2} n \\
& \left.+\frac{20}{\ln 2} \Delta^{2}-\frac{40}{\ln 2} \Delta\right) .
\end{aligned}
$$

This - unfortunately-involves transcendental functions of the variables in an essentially non-algebraic way. By con- sidering the simplified 'complexity' version (1) we have:

$$
\begin{aligned}
d\left(A T^{2}\right) / d \Delta & \equiv d\left\{n \log ^{2} n \cdot 2^{\Delta / 2} /\left(\Delta \log ^{2} \Delta\right)\right\} / d \Delta \\
& =\frac{2^{\Delta / 2}}{\Delta \log ^{2} \Delta} \cdot\left(\frac{\ln 2}{2}-\frac{1}{\Delta}-\frac{2}{\Delta \ln \Delta}\right),
\end{aligned}
$$

which when equated to zero leads to $\ln \Delta(\Delta \ln 2-2)=4$ (again a transcendental equation). This has $\Delta_{\text {optim }}=6$ as integer solution, and because the weights and the thresholds are bounded by $2^{\Delta / 2}$ (Proposition 3) the proof is concluded.

To get a better understanding, the $A T^{2}$ values have been computed for variable fan-ins and different number of inputs $n$, as can be seen in Figure 1, while Figure 2 presents exact plots of the $A T^{2}$ measure which support our previous claim $\Delta_{\text {optim }}=6 . .9$ (as the proof has been obtained using several approximations: neglecting ceilings, using the complexity estimate, etc.).

\section{Size-optimal neural implementations of BFs}

We start from the classical construction developed by Shannon [36] for synthesising one BF with fan-in 2 AND-OR gates. It was extended to the multioutput case and modified to apply to NNs by Horne \& Hush [26]. 
- Proposition 11 (Theorem 3 [26]) Arbitrary Boolean logic functions $f:\{0,1\}^{n} \rightarrow\{0,1\}^{\mu}$ can be implemented in a neural network of perceptrons restricted to fan-in 2 with a node complexity of $\Theta\left\{\mu 2^{n} /(n+\log \mu)\right\}$. The resulting architecture requires $\boldsymbol{O}(n)$ layers.

Sketch of proof The idea is to decompose each output BF into two subfunctions using Shannon's Decomposition [36]:

$f\left(x_{1} x_{2} \ldots x_{n-1} x_{n}\right)=\bar{x}_{1} f_{0}\left(x_{2} \ldots x_{n-1} x_{n}\right)+x_{1} f_{1}\left(x_{2} \ldots x_{n-1} x_{n}\right)$.

By doing this recursively for each subfunction, the output BFs will-in the end-be implemented by binary trees. Horne \& Hush [26] use a trick for eliminating most of the lower level nodes by replacing them with a subnetwork that computes all the possible BF's needed by the higher level nodes. Each subcircuit eliminates one variable and has three nodes (one OR and two ANDs), thus the upper tree has:

$$
\text { size }_{\text {upper }}=3 \mu \cdot \sum_{\substack{n-q-1 \\ i=0}}^{i} 2^{i}=3 \mu\left(2^{n-q}-1\right)
$$

nodes and depth ${ }_{\text {upper }}=2(n-q)$. The subfunctions now depend on only $q$ variables, and a lower subnetwork that computes all the possible BFs of $q$ variables is built. It has:

$$
\text { size }_{\text {lower }}=3 \cdot \sum_{i=1}^{q} 2^{2^{i}}<4 \cdot 2^{2^{q}}
$$

nodes and depth lower $=2 q$ (see Figure 2 from [26]). That $q$ which minimises the size of the two subnetworks is determined by solving $d($ size $) / d q=0$, and gives:

$$
q \approx \log [n+\log \mu-2 \log (n+\log \mu)] .
$$

By substituting this value in (2) and (3), the minimum size:

$$
\text { size } \cong 3 \mu \cdot 2^{n-q} \cong 3 \mu \cdot 2^{n} /(n+\log \mu)
$$

is obtained.

We will use a similar approach for the case when the fan-in is limited by $\Delta$.

Proposition 12 Arbitrary Boolean functions $f:\{0,1\}^{n} \rightarrow$ $\{0,1\}^{\mu}$ can be implemented in a neural network of perceptrons restricted to fan-in $\Delta$ in $O(n / \log \Delta)$ layers.

Proof We use the approach of Horne \& Hush [26] and limit the fan-in to $\Delta$. Each output BF can be decomposed in $2^{\Delta-1}$ subfunctions (i.e., $2^{\Delta-1}$ AND gates). The OR gate would have $2^{\Delta-1}$ inputs. Thus, we have to decompose it in a $\Delta$-ary tree of fan-in $=\Delta$ OR gates. This first decomposition step eliminates $\Delta-1$ variables and generates a tree of:

$$
\begin{gathered}
\text { depth }=1+\lceil(\Delta-1) / \log \Delta\rceil \\
\text { size }=2^{\Delta-1}+\left\lceil\left(2^{\Delta-1}-1\right) /(\Delta-1)\right\rceil .
\end{gathered}
$$

Repeating this procedure recursively $k$ times, we have:

$$
\begin{aligned}
& \text { depth }_{\text {upper }}=k \cdot\{1+\lceil(\Delta-1) / \log \Delta\rceil\} \\
\text { size }_{\text {upper }} & =\left\{2^{\Delta-1}+\left\lceil\left(2^{\Delta-1}-1\right) /(\Delta-1)\right\rceil\right\} \cdot \sum_{i=0}^{k-1} 2^{i(\Delta-1)} \\
& =\text { size } \cdot\left\{2^{k(\Delta-1)}-1\right\} /\left(2^{\Delta-1}-1\right) \\
& \cong 2^{k(\Delta-1)}(1+1 / \Delta) \\
& \approx 2^{k \Delta-k}
\end{aligned}
$$

where the subfunctions depend only on $q=n-k \Delta$ variables. We now generate all the possible subfunctions of $q$ variables with a subnetwork of:

$$
\begin{aligned}
& \text { depth }_{\text {lower }}=[(n-k \Delta) / \Delta\rfloor \cdot\{1+[(\Delta-1) / \log \Delta\rceil\} \\
& \text { size }_{\text {lower }} \\
& =\left\{2^{\Delta-1}+\left\lceil\left(2^{\Delta-1}-1\right) /(\Delta-1)\right\rceil\right\} \cdot \sum^{\ln / \Delta j-k} 2^{2^{n-k \Delta-i \Delta}} \\
& =\operatorname{size} \cdot\left\{2^{2^{0}}+2^{2^{\Delta}}+\ldots+2^{2^{n-(k+1) \Delta}}\right\} \\
& <(\text { size }+1) \cdot 2^{2^{n-(k+1) \Delta}} \\
& \approx 2^{\Delta} \cdot 2^{2^{n-k \Delta-\Delta}}
\end{aligned}
$$

The inequality (7) can be proved by induction. Clearly, size $\cdot 2^{2^{0}}<(s i z e+1) \cdot 2^{2^{0}}$. Let us consider the statement true for $\alpha$; we prove it for $\alpha+1$ :

$$
\begin{aligned}
\text { size } \cdot\left\{2^{2^{0}}+\ldots+2^{2^{\alpha \Delta}}\right\} & + \text { size } \cdot 2^{2^{(\alpha+1) \Delta}} \\
& <\text { size } \cdot 2^{2^{(\alpha+1) \Delta}}+2^{2^{(\alpha+1) \Delta}} \\
\text { size } \cdot\left\{2^{2^{0}}+\ldots+2^{2^{\alpha \Delta}}\right\} & <(\text { size }+1) \cdot 2^{2^{\alpha \Delta}}
\end{aligned}
$$

(due to hypothesis), thus:

$$
(\text { size }+1) \cdot 2^{2^{\alpha \Delta}}<2^{2^{(\alpha+1) \Delta}}
$$

and computing the logarithm of the left side:

$$
\begin{aligned}
2^{\alpha \Delta}+ & \log (\text { size }+1) \\
& =2^{\alpha \Delta}+\log \left\{2^{\Delta-1}+\left\lceil\left(2^{\Delta-1}-1\right) /(\Delta-1)\right\rceil\right\} \\
& <2^{\alpha \Delta}+\log \left\{2^{\Delta-1}+2^{\Delta-1} / \Delta+1\right\} \\
& <2^{\alpha \Delta}+\Delta \\
& <2^{(\alpha+1) \Delta}
\end{aligned}
$$


From (4) and (6) we can estimate depth ${ }_{B F s}$, and from (5) and (8) size $_{B F s}$ as:

$$
\begin{aligned}
\text { depth }_{B F s}= & \{k+\lfloor(n-k \Delta) / \Delta\rfloor\} \cdot\{1+\lceil(\Delta-1) / \log \Delta\rceil\} \\
= & (n / \Delta) \cdot(\Delta / \log \Delta+1) \\
\approx & n / \log \Delta=O(n / \log \Delta) \\
\text { size }_{B F s}= & \mu \cdot \operatorname{size} \cdot\left\{2^{k(\Delta-1)}-1\right\} /(\Delta-1) \\
& +(\text { size }+1) \cdot 2^{2^{n-(k+1) \Delta}} \\
& =\mu \cdot 2^{k \Delta-k}+2^{\Delta} \cdot 2^{2^{n-k \Delta-\Delta}}
\end{aligned}
$$

concluding the proof.

Proposition 13 All the critical points of size ${ }_{B F s}(\mu, n, k, \Delta)$ are relative minimum and are situated in the (close) vicinity of the parabola $k \Delta \approx n-\log (n+\log \mu)$.

Proof To determine the critical points, we equate the partial derivatives to zero. Starting from the approximation (10) of $s i z e_{B F s}$ we compute $\partial s i z e_{B F s} / \partial k=0$ :

$$
\begin{aligned}
& \mu \cdot 2^{k \Delta-k}(\ln 2)(\Delta-1) \\
& +2^{\Delta} \cdot 2^{2^{n-k \Delta-\Delta}}(\ln 2) \cdot 2^{n-k \Delta-\Delta}(\ln 2) \cdot(-\Delta)=0 \\
& \{\mu(\Delta-1) / \Delta /(\ln 2)\} \cdot 2^{2 k \Delta-k-n}=2^{2^{n-k \Delta-\Delta}}
\end{aligned}
$$

and using the notations $k \Delta=\gamma, \beta=\mu(\Delta-1) /(\Delta \ln 2)$, and taking logarithms of both sides:

$$
\log \beta+2 \gamma-k-n=2^{n-\gamma-\Delta}
$$

which has an approximate solution $\gamma \approx n-\log (n+\log \mu)$.

The same result can be obtained by computing with finite differences (instead of approximating the partial derivative):

$$
\begin{gathered}
\text { size }_{B F_{s}}(\mu, n, k+1, \Delta)-\operatorname{size}_{B F s}(\mu, n, k, \Delta)=0 \\
\text { size } \cdot\left\{\mu \cdot 2^{k \Delta-k}-2^{2^{n-k \Delta-\Delta}}\right\}=0 \\
\mu \cdot 2^{k \Delta-k}=2^{2^{n-k \Delta-\Delta}}
\end{gathered}
$$

and after taking twice the logarithm of both sides and using the same notations we have:

$$
\begin{gathered}
\log \{\log \mu+\gamma(1-1 / \Delta)\}=n-\gamma-\Delta \\
\gamma=n-\{\Delta+\log (1-1 / \Delta)\}-\log \{\gamma+\Delta /(\Delta-1) \cdot \log \mu\} \\
\approx n-\Delta-\log (\gamma+\log \mu),
\end{gathered}
$$

which has as the approximate solution:

$$
\gamma=n-\log (n+\log \mu)
$$

Starting again from (10), we compute $\partial \operatorname{size}_{B F s} / \partial \Delta=0$ :

$$
\begin{aligned}
& \mu 2^{k \Delta-k}(\ln 2) k+2^{\Delta}(\ln 2) 2^{2^{n-k \Delta-\Delta}} \\
& +2^{\Delta} 2^{2^{n-k \Delta-\Delta}}(\ln 2) 2^{n-k \Delta-\Delta}(\ln 2)(-k)=0 \\
& \mu k \cdot 2^{\gamma-k}=k(\ln 2) \cdot 2^{n-\gamma} \cdot 2^{2^{n-\gamma-\Delta}}-2^{\Delta} \cdot 2^{2^{n-\gamma-\Delta}} \\
& \mu k \cdot 2^{\gamma-k} \cdot 2^{\gamma-n} \\
& \quad=k(\ln 2) \cdot 2^{2^{n-\gamma-\Delta}}-2^{\Delta} \cdot 2^{\gamma-n} \cdot 2^{2^{n-\gamma-\Delta}} \\
& \mu k \cdot 2^{2 \gamma-k-n} \quad=\left\{k(\ln 2)-2^{\gamma+\Delta-n}\right\} \cdot 2^{2^{n-\gamma-\Delta}}
\end{aligned}
$$

$(\mu / \ln 2) \cdot 2^{2 \gamma-k-n}=\left\{1-2^{\gamma+\Delta-n} /(k \ln 2)\right\} \cdot 2^{2^{n-\gamma-\Delta}}$

which-by neglecting $2^{\gamma+\Delta} /\left\{k(\ln 2) \cdot 2^{n}\right\}$-gives:

$$
\log \beta+2 \gamma-k-n=2^{n-\gamma-\Delta}
$$

i.e., the same equation as (11).

These show that the critical points are situated in the (close) vicinity of the parabola $k \Delta \approx n-\log (n+\log \mu)$.

From Proposition 12 and 13 it follows that size-optimal neural implementations of BFs are obtained for small fanins (i.e., from constant to at most $n-\log n<n$ ). The exact size:

$$
\text { size }_{B F s}=\text { size }_{\text {lower }}+\mu \cdot \text { size }_{\text {upper }}
$$

has been computed for many different values of $n, \mu, \Delta$ and $k$. Some results of those extensive simulations are plotted in Figure 3. From Figure 3(a), (b) and (c) it may seem that $k$ and $\Delta$ used in the proof of Proposition 12 have the same influence on $s_{i z e_{B F S}}$. The discrete parabola-like curves approximating $k \Delta \approx n-\log (n+\log \mu)$ can be seen in Figure $3(d),(e)$ and $(f)$.

Proposition 14 The absolute minimum size ${ }_{B F s}$ is obtained for fan-in $\Delta=2$.

Sketch of proof We will analyse only the critical points by using the approximation $k \Delta \approx n-\log n$. Intuitively the claim can be understood if we replace this value in (10):

$$
\begin{aligned}
\operatorname{size}_{B F S}^{*} & \approx \mu \cdot 2^{n-\log n-k}+2^{\Delta} \cdot 2^{2^{n-n+\log n-\Delta}} \\
& <\mu \cdot 2^{n-\log n}+2^{\Delta} \cdot 2^{2^{\log n}} \\
& =\mu \cdot 2^{n} / n+2^{\Delta} \cdot 2^{n},
\end{aligned}
$$

which clearly is minimised for $\Delta=2$. 

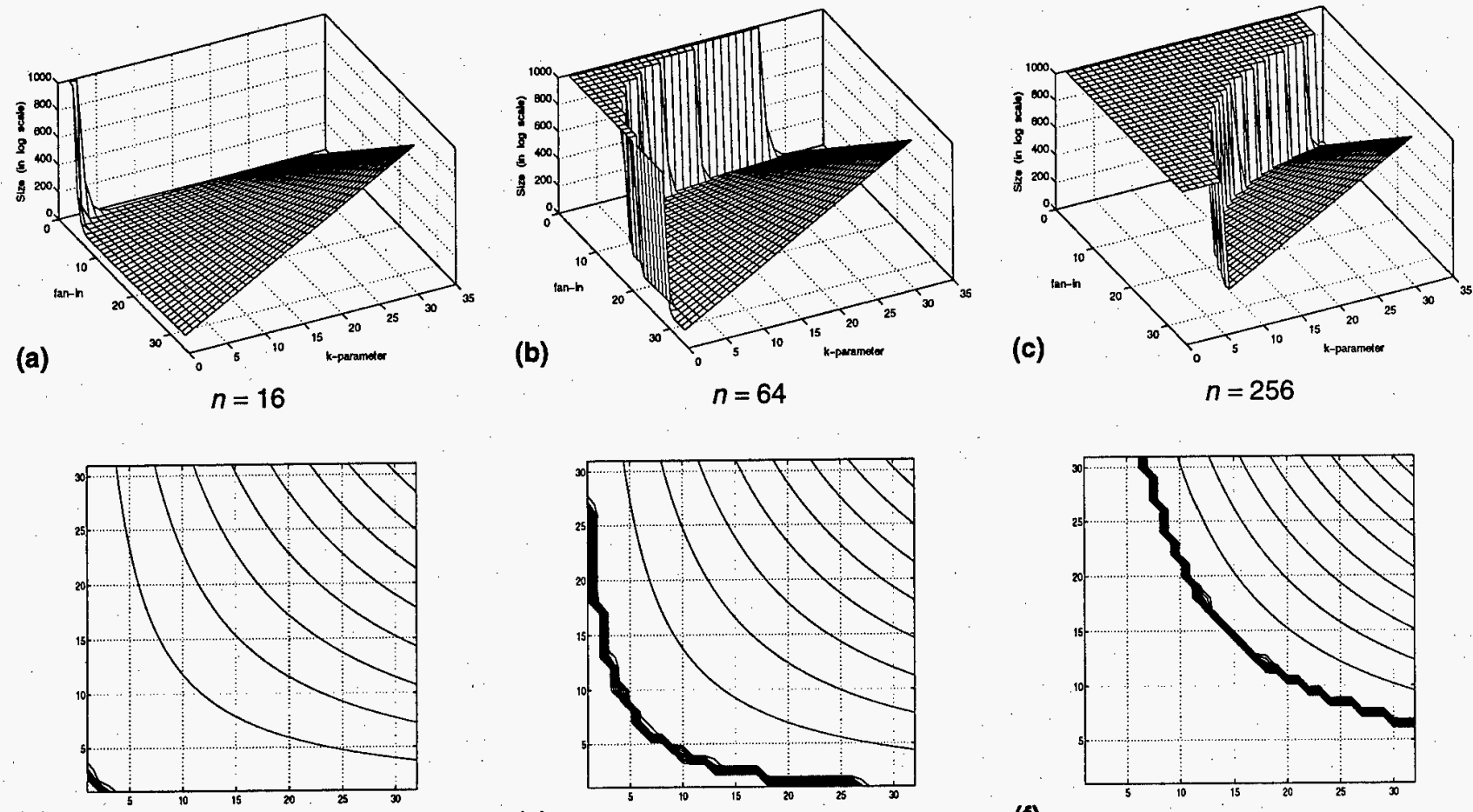

(d)

(e)

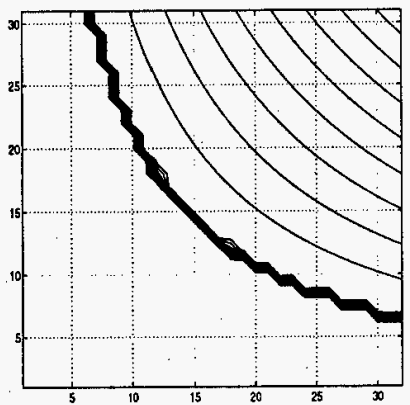

(f)

Figure 3. The size (in logarithmic scale) of NNs implementing arbitrary BFs for: (a) $n=16$; (b) $n=64$; (c) $n=256$ (clipped at $2^{1000}$ ), and the contour plots for the same cases (d), (e), (f).

The detailed proof computes size ${ }_{B F s}(n, \mu, k, \Delta)$ for those $k \approx(n-\log n) / \Delta\left(\right.$ i.e., size $\left._{B F s}^{*}(n, \mu, \Delta)\right)$, and shows that:

$$
\operatorname{size}_{B F s}^{*}(n, \mu, \Delta+1)-\operatorname{size}_{B F s}^{*}(n, \mu, \Delta)>0 .
$$

Hence, the function is monotonically increasing and the minimum is obtained for the smallest fan-in $\Delta=2$. Because the proof has been obtained using successive approximations, several simulation results are presented in Table 3. It can be seen that while for relatively small $n$ the size-optimal solutions are obtained even for $\Delta=16$, starting from $n \geq 64$ all the size-optimal solutions are obtained for $\Delta=2$. It is important that the other relative minima (on, or in the vicinity of the parabola $k \Delta \approx n-\log n$ ) are only slightly larger than the absolute minimum. They might be of practical interest as leading to networks having fewer layers: $n / \log \Delta$ instead of $n$. Last, but not least, it is to be mentioned that all these relative minimum are obtained for fanins strictly lower that linear, as $\Delta \leq n-\log n$.

\section{Size-optimal neural implementations of $I F_{n, m}$ functions}

A similar result can be obtained for $\mathbb{F}_{n, m}$, as the first layer is represented by COMPARISONs (i.e., $I F_{n, 1}$ ) which can be decomposed to satisfy the limited fan-in condition [10, $16,17,21]$.

Table 3

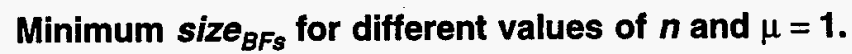

\begin{tabular}{|c|c|c|c|c|c|c|c|c|c|}
\hline$n$ & $8=2^{3}$ & $16=2^{4}$ & $32=2^{5}$ & $64=2^{6}$ & $128=2^{7}$ & $256=2^{8}$ & $512=2^{9}$ & $1024=2^{10}$ & $2048=2^{11}$ \\
\hline \hline size & 110 & 1470 & 349,530 & $1.611 \times 10^{9}$ & $6.917 \times 10^{18}$ & $5.104 \times 10^{38}$ & $2.171 \times 10^{76}$ & $1.005 \times 10^{154}$ & $1.685 \times 10^{307}$ \\
\hline$\Delta$ & 4 & 8 & 16 & 2 & 2 & 2 & 2 & 2 & 2 \\
\hline$k \Delta$ & 4 & 8 & 16 & 58 & 122 & 248 & 504 & 1014 & 2038 \\
\hline
\end{tabular}



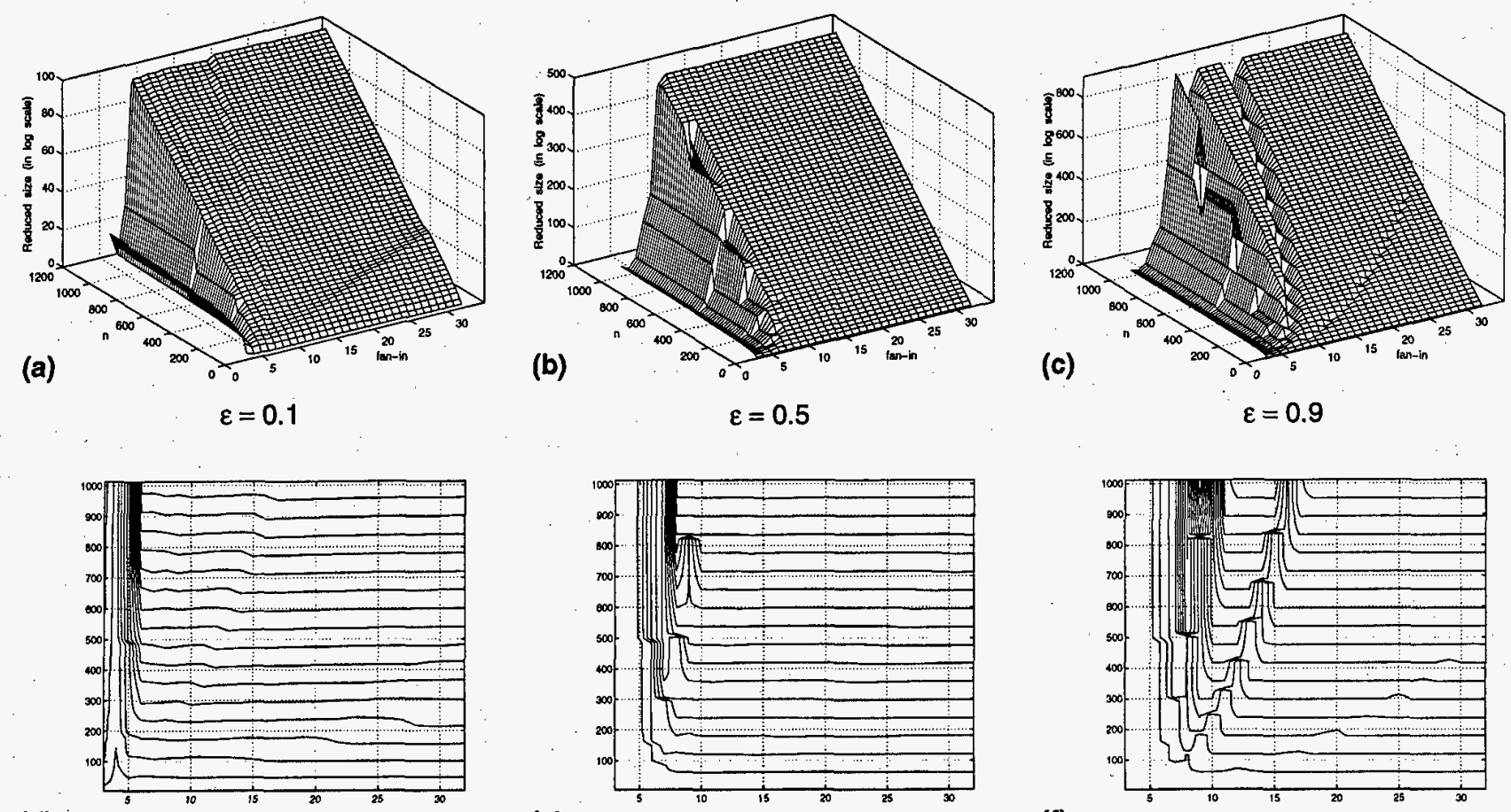

(d)

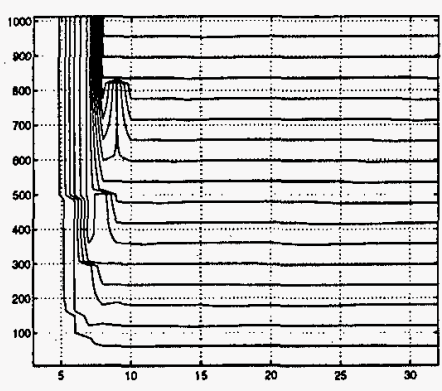

(e)

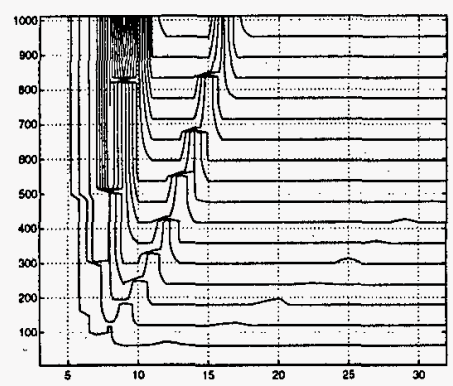

(f)

Figure 4. The reduced size (in logarithmic scale) of NNs implementing $I F_{n, m}$ functions for $m=2^{\varepsilon n}$ : (a) $\varepsilon=0.1$; (b) $\varepsilon=0.5$; (c) $\varepsilon=0.9$; and the contour plots for the same cases (d), (e), (f). The lowest values are obtained for very small constant fan-in values.

Proposition 15 (Lemma 1 \& Corollary 1 [21]) The COMPARISON of two n-bit numbers can be computed by a $\Delta$-ary tree neural network having integer weights and thresholds bounded by $2^{\Delta / 2}$ for any $3 \leq \Delta \leq n$.

The size complexity of the $\mathrm{NN}$ implementing one $\boldsymbol{F}_{n, m}$ function is [21]:

$$
\text { size }_{B_{-} \Delta}=2 n m \cdot\left\{\frac{1}{\Delta / 2}+\ldots+\frac{1}{(\Delta / 2)^{\text {depth }_{B_{-} \Delta}}}\right\} \text {, }
$$

where depth ${ }_{\mathrm{B} \Delta \Delta}=\lceil\log n /(\log \Delta-1)\rceil$, but a substantial enhancement is obtained if the fan-in is limited. Due to the limitation, the maximum number of different $B F s$ which can be computed in each layer is:

$$
\begin{aligned}
(2 n / \Delta) \cdot 2^{\Delta}, \quad \frac{2 n / \Delta}{\Delta / 2} \cdot 2^{\Delta(\Delta / 2)}, \ldots, & \frac{2 n / \Delta}{(\Delta / 2)^{\text {depth }_{B-1}-1}} \cdot 2^{\Delta(\Delta / 2)^{\text {depth }_{B-\Delta}-1}} .
\end{aligned}
$$

For large enough $m$ (needed for achieving a certain precision $[10,23,42])$, and/or large enough $n$, the first terms of the sum (13) will be larger than the equivalent ones from (14). This is equivalent to the trick from [26], as the lower levels will compute all the possible functions using only limited fan-in COMPARISONs. Hence, the optimum size becomes:

$$
\text { size }_{\mathbf{B}_{\Delta} \Delta}^{\text {optim }}=2 n \cdot\left\{\sum_{i=1}^{k} \frac{2^{\Delta(\Delta / 2)^{i-1}}}{\Delta(\Delta / 2)^{i-1}}+\sum_{i=k+1}^{\text {depth } \Delta .} \frac{m}{(\Delta / 2)^{i}}\right\}
$$

explained as the same BFs are computed redundantly. In terms of fan-in, several exponentially decreasing terms will be replaced by double exponential increasing terms.

Following similar steps to the ones used in Proposition 13 , it is possible to show that the minimum size is obtained for $\Delta=3$. To get a better understanding we have done extensive simulations by considering that $m=2^{\varepsilon n}$. Some of the results of these simulations can be seen in Figure 4 . They show that it is always possible to obtain a significant reduction of the size by properly choosing a small constant fan-in. It is to be mentioned that the size reduction is by a huge factor which is of the form $2^{\varepsilon n-c}$ for very small fanins $\Delta_{\text {optim }}=4$...6. 


\section{Conclusions and further work}

The paper has focused on sparsely connected NNs, i.e. having either (small) constant fan-ins, or at most logarithmic in the number of inputs $n$. Using different cost functions-which are closer estimates than size and depth for the area and the delay of a VLSI chip-we have been able to prove that VLSI-optimal implementations of $\boldsymbol{F}_{n, m}$ functions are obtained by small constant fan-ins.

Concerning size-optimal solutions, we have shown that:

- arbitrary BFs require small-but not necessarily constant-fan-ins (at most $n-\log n$ );

- $F_{n, m}$ functions require small constant fan-ins.

Some of these results have already been applied to optimising the VLSI design of a neural constructive algorithm $[5,14,15]$. We are working on a mixed constructive algorithm which-after quantizing the input space as in $[4,11$, $12,13]$ - could synthesise $\mathbb{F}_{n, m}$ functions, arbitrary BFs, or a mixture of them such as to reduce the area of the resulting VLSI chip. This could also have applications to automatic synthesis of mixed analog/digital circuits $[8,16]$. An alternative solution currently under investigation [14] is to use such a synthesis step after quantizing the input space as detailed in [24].

Future work should concentrate on:

- linking such results with the 'Occam's razor' [44] and the 'minimum description length' [43];

- finding closer estimates (i.e., other cost functions) for optimal mixed analogue/digital implementations.

The main conclusion is that VLSI-optimal solutions can be obtained for small constant fan-ins. We mention here that there are similar small constants relating to our capacity of processing information [31].

\section{References}

[1] Y.S. Abu-Mostafa, "Connectivity Versus Entropy," in D.Z. Anderson (ed.): Neural Info. Proc. Sys., Amer. Inst. of Physics, NY, 1-8, 1988.

[2] N. Alon \& J. Bruck, "Explicit Construction of Depth-2 Majority Circuits for Comparison and Addition," Tech. Rep. RJ 8300 (75661), IBM Almaden, San Jose, CA, 1991.

[3] P.L. Bartlett, "The Sample Complexity of Pattern Classification with Neural Networks: The Size of the Weights Is More Important than the Size of the Network," Tech. Rep. (ftp:syseng.anu.edu. au/pub/peter/TR96d.ps.Z), Dept. Sys. Eng., Sch. of Info. Sci. and Eng., Australian National Univ., Canberra 0200 Australia, May 1996 (short version in M.C. Mozer, M.I. Jordan \& T. Petsche (eds.): Advances in Neural Info. Proc. Sys. 9, MIT Press, MA, 1997).

[4] V. Beiu, "Entropy Bounds for Classification Algorithms," Neural Network World, 6(4), 497-505, 1996.

[5] V. Beiu, "Optimal VLSI Implementation of Neural Net- works," in J.G. Taylor (ed.): Neural Networks and Their Applications, John Wiley, Chichester, 255-276, June 1996.

[6] V. Beiu, "VLSI Complexity of Threshold Gate COMPARIson," Conf. Rep. Intl. Symp. on Neuro-Fuzzy Sys. (AT'96, Lausanne, Switzerland), Adv. \& Appls. Tech. Inst. (Aati), Ecubens, 161-170, August 1996.

[7] V. Beiu, "New VLSI Complexity Results for Threshold Gate COMPARISON," in T.B. Ludermir (ed.): Proc. 3rd Brazilian Symp. on Neural Networks (III SBRN, Recife, Brazil), UFPE-DI, Recife, 251-258, November 1996.

[8] V. Beiu, "Optimization of Circuits Using a Constructive Learning Algorithm," Tech. Rep. LA-UR-97-851, Los Alamos Natl. Lab., USA; in A.B. Bulsari \& S. Kallio (eds.): Neural Networks in Engineering Systems (EANN'97, Stockholm, Sweden), Systems Engineering Association, Turku, Finland, 291-294, June 1997.

[9] V. Beiu, "On the Circuit and VLSI Complexity of Threshold Gate COMPARISON," Tech. Rep. LA-UR-96-3591, Los Alamos Natl. Lab., USA, Dec. 1996; to appear in Neurocomputing, 1997/8.

[10] V. Beiu, VLSI Complexity of Discrete Neural Networks, Gordon and Breach, Newark, NJ, 1998.

[11] V. Beiu \& T. De Pauw, "Tight Bounds on the Size of Neural Networks for Classification Problems," Tech. Rep. LAUR-97-60, Los Alamos Natl. Lab., USA; in J. Mira, R. Moreno-Díaz \& J. Cabestany (eds.): Biological and Artificial Computation: From Neuroscience to Technology (IWANN'97, Lanzarote, Spain), Lecture Notes in Comp. Sci., vol. 1240, Springer, Berlin, 743-752, June 1997.

[12] V. Beiu \& S. Draghici, "Limited Weights Neural Networks: Very Tight Entropy Based Bounds," Tech. Rep. LAUR-97-294, Los Alamos Natl. Lab., USA; in D.W. Pearson (ed.): Proc. Intl. ICSC Symp. on Soft Computing (SOCO '97, Nîmes, France), ICSC Academic Press, Canada, 111118, September 1997.

[13] V. Beiu \& H.E. Makaruk, “Computing $n$-Dimensional Volumes of Complexes: Application to Constructive Entropy Bounds," Tech. Rep. LA-UR-97-2873, Los Alamos Natl. Lab., USA; to appear in Proc. Intl. Symp. on Nonlinear Theory and Its Applications (NOLTA'97, Hawaii, USA), November 29 - December 3, 1997.

[14] S. Draghici, V. Beiu \& I.K. Sethi, "A VLSI Optimal Constructive Algorithm for Classification Problems," Tech. Rep. LA-UR-97-1609, Los Alamos Natl. Lab., USA; to appear in C.H. Dagli, M. Akay, O. Ersoy, B. Fernández \& A. Smith (eds.): Smart Engineering System Design: Neural Networks, Fuzzy Logic, and Evolutionary Programming (ANNIE'97, St. Louis, USA), November 9-12, ASME Press, Fairfield, NJ, 1997.

[15] V. Beiu \& J.G. Taylor, "VLSI Optimal Neural Network Learning Algorithm," in D.W. Pearson, N.C. Steele \& R.F. Albrecht (eds.): Artif. Neural Nets \& Genetic Algorithms (ICANNGA'95, Alès, France), Springer-Verlag, Vienna, 61-64, 1995.

[16] V. Beiu \& J.G. Taylor, "Direct Synthesis of Neural Networks," Proc. MicroNeuro'96 (Lausanne, Switzerland), IEEE CS Press, Los Alamitos, CA, 257-264, 1996. 
- [17] V. Beiu \& J.G. Taylor, "On the Circuit Complexity of Sigmoid Feedforward Neural Networks," Neural Networks, 9(7), 1155-1171, 1996.

[18] V. Beiu, J.A. Peperstraete, J. Vandewalle \& R. Lauwereins, "Efficient Decomposition of COMPARISON and Its Applications," in M. Verleysen (ed.): ESANN'93 (Brussels, Belgium), Dfacto, Brussels, 45-50, 1993.

[19] V. Beiu, J.A. Peperstraete, J. Vandewalle \& R. Lauwereins, "COMPARISON and Threshold Gate Decomposition," in D.J. Myers \& A.F. Murray (eds.): MicroNeuro'93 (Edinburgh, UK), UnivEd Tech. Ltd., Edinburgh, 83-90, 1993.

[20] V. Beiu, J.A. Peperstraete, J. Vandewalle \& R. Lauwereins, "Learning from Examples and VLSI Implementation of Neural Networks," in R. Trappl (ed.): Cybernetics \& Sys. Res.'94 (EMCSR'94, Vienna, Austria), World Scientific, Singapore, 1767-1774, 1994.

[21] V. Beiu, J.A. Peperstraete, J. Vandewalle \& R. Lauwereins, "Area-Time Performances of Some Neural Computations," in P. Borne, T. Fukuda \& S.G. Tzafestas (eds.): SPRANN '94 (Lille, France), GERF EC, Lille, 664-668, 1994.

[22] J. Bruck \& J.W. Goodmann, "On the Power of Neural Networks for Solving Hard Problems," in D.Z. Anderson, (ed.): Neural Info. Proc. Sys., Amer. Inst. of Physics, NY, 137-143, 1988; also in J. Complexity, 6, 129-135, 1990.

[23] J.S. Denker \& B.S. Wittner, "Network Generality, Training Required and Precision Required," in D.Z. Anderson (ed.): Neural Info. Proc. Sys., Amer. Inst. of Physics, NY, 219$222,1988$.

[24] S. Draghici \& I.K. Sethi, "On the Possibilities of the Limited Precision Weights Neural Networks in Classification Problems," in J. Mira, R. Moreno-Díaz \& J. Cabestany (eds.): Biological and Artificial Computation: From Neuroscience to Technology (IWANN'97, Lanzarote, Spain), Lecture Notes in Comp. Sci., vol. 1240, Springer, Berlin, 753-762, 1997.

[25] D. Hammerstrom, "The Connectivity Analysis of Simple Association -or- How Many Connections Do You Need," in D.Z. Anderson (ed.): Neural Info. Proc. Sys., Amer. Inst. of Physics, NY, 338-347, 1988.

[26] . B.G. Horne \& D.R. Hush, "On the Node Complexity of Neural Networks," Neural Networks, 7(9), 1413-1426, 1994.

[27] S. Hu, Threshold Logic, Univ. California Press, Berkeley, CA, 1965.

[28] H. Klaggers \& M. Soegtrop, "Limited Fan-in Random Wired Cascade-Correlation," in D.J. Myers \& A.F. Murray (eds.): MicroNeuro'93 (Edinburgh, UK), UnivEd Tech. Ltd., Edinburgh, 79-82, 1993.

[29] A.V. Krishnamoorthy, R. Paturi, M. Blume, G.D. Linden, L.H. Linden \& S.C. Esener, "Hardware Tradeoffs for Boolean Concept Learning," Proc. WCNN'94 (San Diego,
USA), Lawrence Erlbaum \& INNS Press, Hillsdale, CA, 551-559, 1994.

[30] R.D. Mason \& W. Robertson, "Mapping Hierarchical Neural Networks to VLSI Hardware," Neural Networks, 8(6), 905-913, 1995.

[31] G.A. Miller, "The Magical Number Seven, Plus or Minus Two: Some Limits on our Capacity for Processing Information", Psych. Rev., 63(1), 71-97, 1956.

[32] R.C. Minnik, "Linear-Input Logic," IRE Trans. on Electr. Comp., 10(1), 6-16, 1961.

[33] D.S. Phatak \& I. Koren, "Connectivity and Performances Tradeoffs in the Cascade Correlation Learning Architecture," IEEE Trans. Neural Networks, 5(6), 930-935, 1994.

[34] N.P. Red'kin, "Synthesis of Threshold Circuits for Certain Classes of Boolean Functions," Kibernetika 5(1), 6-9, 1970; English translation in Cybernetics 6(5), 540-544, 1973.

[35] V.P. Roychowdhury, A. Orlitsky \& K.-Y. Siu, "Lower Bounds on Threshold and Related Circuits via Communication Complexity," IEEE Trans. Info. Th., 40(2), 467-474, 1994.

[36] C. Shannon, "The Synthesis of Two-Terminal Switching Circuits," Bell Sys. Tech. J., 28(1), 59-98, 1949.

[37] K.-Y. Siu, V.P. Roychowdhury \& T. Kailath, "Depth-Size Tradeoffs for Neural Computations," IEEE Trans. Comp., 40(12), 1402-1412, 1991.

[38] J.D. Ullman, Computational Aspects of VLSI, Comp. Sci. Press, Rockville, MA, 1984.

[39] S. Vassiliadis, S. Cotofana \& K. Berteles, "2-1 Addition and Related Arithmetic Operations with Threshold Logic," IEEE Trans. Comp., 45(9), 1062-1068, 1996.

[40] M.R. Walker, S. Haghighi, A. Afghan \& L.A. Akers, "Training a Limited-Interconnect, Synthetic Neural IC," in D.S. Touretzky (ed.): Advances in Neural Info. Proc. Sys. 1, Morgan Kaufmann, San Mateo, CA, 777-784, 1989.

[41] R.C. Williamson, " $\varepsilon$-Entropy and the Complexity of Feedforward Neural Networks," in R.P. Lippmann, J.E. Moody \& D.S. Touretzky (eds.): Advances in Neural Info. Proc. Sys. 3, Morgan Kaufmann, San Mateo, CA, 946-952, 1990

[42] J. Wray \& G.G.R. Green, "Neural Networks, Approximation Theory, and Finite Precision Computation," Neural Networks, 8(1), 31-37, 1995.

[43] R.S. Zemel, "Minimum Description Length Analysis," in M.A. Arbib (ed.): The Handbook of Brain Theory and Neural Networks, MIT Press, Cambridge, MA, 572-575, 1995.

[44] B.-T. Zhang \& H. Mühlenbein, "Genetic Programming of Minimal Neural Networks Using Occam's Razor," Tech. Rep. GMD 734, Schloß Birlinghoven, Sankt Augustin, Germany, 1993 (also in Complex System, 7(3), 199-220, 1993). 
M98004666

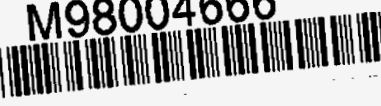

Report Number (14) LA -UR $-997-4314$ CONF-921235--

Publ. Date (11) 199803

Sponsor Code (18) DOELHR, XE

UC Category (19) UC-905, DOE/ER

DOE 